\title{
Modèles et réalités incertaines de l'Université
}

\section{Lucia Granget}

\section{OpenEdition}

Journals

Édition électronique

URL : http://journals.openedition.org/communicationorganisation/3469

DOI : 10.4000/communicationorganisation.3469

ISSN : 1775-3546

\section{Éditeur}

Presses universitaires de Bordeaux

\section{Édition imprimée}

Date de publication : 1 octobre 2006

Pagination : 164-186

ISSN : 1168-5549

\section{Référence électronique}

Lucia Granget, « Modèles et réalités incertaines de l'Université », Communication et organisation [En

ligne], 30 | 2006, mis en ligne le 21 juin 2012, consulté le 30 avril 2019. URL : http://

journals.openedition.org/communicationorganisation/3469; DOI : 10.4000/

communicationorganisation.3469

Ce document a été généré automatiquement le 30 avril 2019

(C) Presses universitaires de Bordeaux 


\title{
Modèles et réalités incertaines de l'Université
}

\author{
Lucia Granget
}

Kuan Tzu (Confucius) déclarait, il y a vingt-six

siècles :

«Si tu projettes ta pensée un an à l'avance, plante des graines,

Si tu projettes ta pensée dix ans à l'avance, plante

un arbre,

Si tu projettes ta pensée cent ans à l'avance, enseigne. »

1 La signification de l'université est en train de changer. Sa forme, ses contenus et ses objectifs deviennent flous. La société mondiale s'organise de plus en plus autour de la production des savoirs. Vecteur de l'innovation et moteur de l'économie, le savoir est envisagé comme un marché. A la fois, source et bénéficiaire du changement, l'université évolue dans un contexte à faible prévisibilité alors que ses activités principales, la formation et la recherche, correspondent au temps long. Les conséquences des décisions prises à un moment et en fonction d'une conjoncture donnée, se produiront dans un monde très différent de celui pour lequel, elles ont été envisagées.

2 L'idée de crise, si représentative de notre système d'enseignement supérieur semble finalement assez rassurante. Elle renvoie uniquement à la modification provisoire d'un état, avec un système qui devrait retrouver sa stabilité. Mais dans un univers où tout se transforme, il nous a semblé pertinent de revisiter les modèles organisationnels appliqués à l'université selon les théories des organisations et du management et de les confronter aux nombreux discours actuels. Face à la montée en puissance de l'université « entrepreneuriale », nous nous interrogerons sur les contradictions qu'un tel modèle ne manque pas de susciter, notamment au travers de l'introduction depuis une vingtaine d'années de la communication stratégique. Nous tenterons de définir la place qu'occupe 
le marketing dans une organisation éloignée de la rationalité parfois supposée « objective » et souvent caractérisée par le fameux « modèle de l'anarchie organisée ».

Notre démarche combine plusieurs approches. Elle s'appuie sur un corpus d'ouvrages et d'articles portant sur l'étude de l'établissement universitaire et de son gouvernement. Des rapports récents traitant du développement de l'enseignement supérieur français complètent l'analyse. A cette première approche, sont associés les résultats partiels d'une « recherche-action ». Deux séries d'entretiens semi-directifs ont été menées, auprès d'une vingtaine de responsables de communication, à intervalle de deux ans (2002 et 2004). L'objectif était de cerner les perceptions individuelles et les construits utilisés par les interviewés. Le recueil des opinions et des croyances a permis de mieux comprendre la façon dont évolue la communication des universités dans un contexte de changement. La seconde enquête (2004) a permis d'éveiller chez les interviewés une analyse plus sévère et davantage embarrassée sur les pratiques actuelles. Elle a mis en évidence les questions que se posent les responsables de communication. L'analyse des documents de communication (depuis 1998) complète le dispositif. Le recours à l'analyse de contenu par comptage de certains mots ou phrases et l'étude de leurs fréquences sur une période de sept ans a favorisé la mise en avant de l'émergence d'un discours largement emprunté à l'entreprise. Au cours de cette période, il a été noté une importante diversification des outils et une amélioration notable de la qualité esthétique des documents de communication. Toutefois, il convient de préciser au moins trois principales limites. Le travail d'enquête a porté exclusivement sur une catégorie d'acteurs universitaires, considérée comme émetteur principal. Cette limite est importante car il faut bien reconnaître que différentes entités (UFR, services, laboratoires de recherche) développent leur propre politique de communication. Les écoles ont été exclues de l'enquête, ce qui ne permet pas de confirmer l'influence qu'elles sont supposées exercer. Ont été également évincées les universités étrangères (notamment européennes et américaines) susceptibles de créer un effet de mimétisme.

\section{Les modèles organisationnels de l'université selon les théories des organisations et du management}

\section{Présentation générale}

Dans les années 60, émergent les premiers travaux sur le gouvernement des universités. La littérature anglo-saxone définit l'université comme une organisation "collégiale " dont les décisions sont consensuelles et prises en interne entre pairs. Selon ce modèle la communauté universitaire s'auto organise sans prendre en compte l'autorité hiérarchique extérieure (Goodman, 1962, Millett, 1962). Ce modèle fera l'objet de critiques car il néglige la dimension politique inhérente à la prise de décision. Toutefois la collégialité demeure une caractéristique culturelle des universités pour de nombreux auteurs (Dill et Sporn, 1995, Currie et Vidovitch, 1998). Pour Baldridge (1971), les conflits entre les facultés entraînent des marchandages. La répartition des ressources budgétaires en interne notamment dans un contexte de pénurie (Pfeffer et Salancik, 1974) a permis de mettre en évidence le modèle "politique ». Les facultés qui bénéficient d'une bonne notoriété en externe vont être en capacité de la faire valoir au moment des négociations budgétaires en interne. La rationalité de cette vision fera l'objet à son tour de travaux critiques. Le modèle de «l'anarchie organisée », développé par Cohen et March (1974) 
décrit une absence de cohérence généralisée (objectifs et missions multiples, processus de production relevant d'une technologie complexe, participation fluctuante des membres). Les «systèmes faiblement liés» de Weick (1976) mettent également en évidence des organisations universitaires caractérisées par des manques de coordinations et de régulations, des liens faibles entre le personnel administratif et les enseignants, une inadaptation de la structure à l'activité, une absence d'homogénéité entre les différents départements avec des objectifs, des missions, des méthodes de gestion qui entraînent d'une part, des cloisonnements et d'autre part, un manque de transparence. Dans les années 1990, en raison des nombreux changements qui affectent l'université, une nouvelle conception voit le jour dont le modèle le plus cité est celui du « triangle de Clark »(1983). Les trois sommets de ce triangle représentent, l'Etat, le marché et les oligarchies universitaires. Ce modèle sera discuté et amélioré par de nombreux auteurs et par Clark lui-même.

5 L'idée d'université « entrepreneuriale » émerge, mais elle est confrontée à de nombreux obstacles. La massification de l'enseignement supérieur, à partir de 1945, avait provoqué une inévitable bureaucratisation. Le modèle bureaucratique de Weber (1971) repose sur un pouvoir "légal-rationnel " dont le mode d'organisation structuré, réunit «les conditions d'administration et de production de masse ». Il correspond au rationalisme occidental moderne. Les travaux américains et notamment ceux de Merton (1957), Gouldner (1954), March et Simon (1958) ont remis en cause le modèle bureaucratique. Crozier (1963) montrera notamment que l'individu reste autonome par rapport à l'organisation bureaucratique et qu'il est capable de développer des stratégies pour se protéger ou améliorer sa position à l'intérieur du système.

6 L'administration dans son ensemble est marquée par son immobilisme et ses dysfonctionnements. Des blocages et des comportements qui visent à détourner des règles nombreuses et contraignantes rendent complexes les décisions malgré l'introduction dans les années 70-80, de nouveaux outils de gestion publique. Mais l'image d'une «société bloquée » (Crozier) demeure persistante. Le modèle de l'entreprise dans les années 80 , semble pourtant en mesure de pourvoir régler tous les problèmes.

\section{De « l'anarchie organisée » au « modèle de la poubelle »}

7 Les travaux de Michael Cohen, James G. March et Johan Olsen interviennent à la fin des années 60 , au cours d'une période fortement marquée par les mouvements étudiants. Les auteurs élaborent deux concepts-clés qui visent à comprendre les processus de décisions dans les anarchies organisées. L'université constitue un exemple de ce type d'organisation. Elle est une institution dont les objectifs ne sont pas toujours cohérents. Ils ne sont pas partagés par tous les acteurs dont les intérêts peuvent être divergents. Les lieux de décisions sont multiples et les objectifs sont parfois contradictoires. Les processus de production sont complexes et la technologie est mal maitrisée.

8 Le premier concept est basé sur le constat suivant : « Il y a de l'ordre dans le monde, mais cet ordre ne correspond pas à celui que l'on attend " (March, 2005). L'organisation est conceptualisée comme un flot de poubelles correspondant au processus de décisions. La décision n'est pas dans le modèle de la poubelle le résultat d'une séquence dictée par la rationalité (identification des objectifs, étude des solutions disponibles et de leurs 
conséquences) ou par la confrontation entre objectifs, solutions et conséquences selon le modèle politique. Au contraire, il existe des solutions qui peuvent résoudre des problèmes (le choix de la réponse intervient souvent en amont de l'examen du problème à résoudre). La décision provient de la rencontre fortuite entre solutions et problèmes. Chaque occasion de choix correspond à une poubelle dans laquelle sont jetés les solutions et les problèmes en fonction de leur ordre d'apparition. Si un problème survient, c'est l'occasion pour les acteurs d'affirmer une opinion préalable à l'occasion de la prise de décision. Les organisations ne sont donc plus des agents rationnels obéissants à une planification.

9 Le second concept met en évidence des individus qui agissent de façon simultanée. Les prises de décisions dépendront alors des participants présents (chacun ayant la possibilité d'intervenir ou au contraire de rester passif), « ceux qui n'ont rien de mieux à faire à ce moment-là » (Rojot, 2005). « En conséquence, les décisions prises dépendent beaucoup des flux d'arrivée des problèmes, des solutions... et des participants » (March, 2005). Le leadership ne peut être que faiblement directif. C'est le modèle de l'anarchie organisée qui va définir implicitement le rôle du président d'université. On retrouve cette vision de la dimension symbolique du leadership universitaire dans des travaux plus récents (Birnbaum, 1988, Tierney, 1989, Middlehurst, 1995).

Christine Musselin (1989) critiquera le modèle de l'anarchie organisée appliqué aux universités et notamment (1997) la relation systématique entre anarchie organisée et modèle décisionnel de la poubelle. Pour Musselin, l'étude du processus de décision dans les universités permet d'identifier des permanences et des régularités non aléatoires. Dans «La longue marche des universités françaises " (2001), l'auteur identifie un tournant majeur: le lancement de la politique de contractualisation en 1989. Cette politique a permis de minimiser et de marginaliser les références disciplinaires, de générer une "sorte de doctrine de l'autonomie des universités ", de permettre "une nouvelle représentation de l'université ", en favorisant "une certaine prise en charge de leur gestion, tout en sécrétant une solidarité technique ». Musselin constate également, que le processus de réflexion nécessaire à la préparation du projet de l'université et la négociation avec la tutelle, sont devenus progressivement plus important que le contenu du contrat. Stéphanie Mignot-Gérard (2003) montre par ailleurs, que «le leadership qui s'exerce au sein des organisations universitaires est éclaté entre plusieurs acteurs dont les intérêts ne sont pas forcément concordants ». Elle conclut en constatant que «finalement, le gouvernement d'une université est le produit de cet assemblage relationnel complexe : la relation entre les différents leaders, la relation entre les leaders et les instances, et l'interdépendance entre les différentes instances ».

11 Ces deux approches tendent à montrer une tendance à la prise de décision à la fois collégiale et politique. La gestion de la dimension relationnelle semble alors en capacité de supplanter la rationalité des techniques et des outils avec pour limite sans doute, des dispositions naturelles relationnelles inégalement réparties entre les individus. Ainsi, la décision est le résultat d'un jeux d'influence faisant intervenir divers paramètres: valeurs, compétences relationnelles, désirs, rationalités, facteurs cognitifs. L'illusion de la rationalité de la décision a été largement démontrée dans les services publics et dans les entreprises. Lucien Sfez (1981) affirme même que la décision au sens de temps $t$ de la délibération n'existe pas. March (2005) rappelle qu'à l'origine, le modèle du garbage can était organisé. Les «gens qui se sont emparés du modèle de la poubelle l'ont fait d'une façon métaphorique. Ils ont mis l'accent sur l'aspect anarchique plus que sur la 
cohérence, y voyant là une bonne illustration de toutes sortes d'organisations désorganisées ».

\section{L'université entrepreneuriale}

12 Les travaux menés au cours de ces vingt dernières années tendent à démontrer que les universités sont de plus en plus conquises par les méthodes issues du management des entreprises. Le modèle de Clark (1983) développe une typologie des universités des années 60 et 70 . Le triangle de coordination permet de mettre en évidence pour les universités américaines, une coordination -intégration par le marché, pour les universités soviétiques et suédoises, par l'Etat, ainsi que la mise en évidence d'oligarchies, pour l'Italie et le Royaume-Uni. En 1998, Clark rajoutera une nouvelle dimension à son modèle "hierarchical sef-guidance of university leaders " en réponse à un fort développement de l'université entrepreneuriale en raison notamment d'une baisse des fonds publics, de la nécessaire adaptation à de nouveaux publics, au marché du travail et à l'industrie de la connaissance. La gouvernance de l'université invite à plus de flexibilité et de rapidité pour répondre au changement de l'environnement. Des comportements d'entrepreneurs et une modification des valeurs s'imposent pour que la culture entrepreneuriale soit diffusée dans toutes les parties de l'université. L'association de plusieurs facteurs est alors nécessaire : un élargissement des unités d'enseignement et de recherche avec dépassement des frontières disciplinaires, une valorisation de la recherche et de l'offre de formation, une diversification des sources de financement, une association des enseignants-chercheurs aux projets interdisciplinaires et au pilotage de l'université dans le but de faire accepter un mode de direction plus managérial. Slaugter et Leslie en 1997 vont montrer comment la place traditionnelle de l'université entre travail et capital est modifiée par la globalisation de l'économie. De nouvelles sources de financement sont recherchées (recherches appliquées, droits d'inscription). Les dirigeants des universités sont comparés à des entrepreneurs subventionnés. Clark (1996) met en évidence la complexification du système confrontée à une logique de différenciation qui échappe à l'autorité bureaucratique pour pouvoir s'adapter au marché. De l'ensemble de ces travaux émergent un questionnement commun : comment parvenir à transformer le modèle organisationnel de l'université, marqué par une capacité de résistance aux changements malgré la pression de l'environnement, pour parvenir à un système entrepreneurial ?

13 La transformation du système universitaire mis en évidence par ces auteurs s'inscrit dans une perspective profonde et durable. Les mécanismes qui régissent son organisation ont changé de sens au cours de l'histoire. Le processus de Bologne n'est que la partie la plus visible. Il est le résultat d'une forme de consensus qui vise à maximiser la contribution de l'enseignement supérieur et de la recherche à la compétitivité économique. Mais l'idée d'envisager l'adéquation entre formations et besoins de l'économie n'est pas récente. Dès le milieu du XIXe siècle, Marx insistait sur l'invention comme activité économique. L'application directe de la science à la production est un critère déterminé et requis par la production. Mais avant d'être au service de l'entreprise, les autorités publiques, au cours des XIVème et $\mathrm{XV}^{\text {ème }}$ siècle, s'intéressent aux universités pour apporter une réponse aux besoins d'une administration en plein développement. Au XVIII ème, les réformes universitaires se succèdent en faveur "d'une adéquation plus étroite aux besoins des Etats et des professions »(Charle, Verger, 1994). Entre 1806 et 1808, Napoléon crée 
l'université Impériale qui limite l'enseignement supérieur à sa fonction utilitariste. Au cours de la période 1860-1940, les enjeux sont: «la promotion sociale des individus, l'affirmation nationale, le progrès scientifique et économique national et international, la formation des élites et (...), l'évolution du rapport entre les sexes (...)» (Charle, Verger, 1994). Au cours de cette période, le système universitaire américain s'affirme en raison de l'industrialisation et de l'urbanisation. Il laisse une place croissante à la recherche.

Depuis 1945, la demande de formation est exponentielle partout dans le monde alors que les modes d'organisation ont été conçus pour des effectifs réduits. Par ailleurs, l'objectif des sociétés contemporaines est désormais focalisé sur la science et ses applications techniques, militaires et économiques. Dès 1944, Wiener et quelques scientifiques protestent contre la soumission de la science à l'establishment militaire. Heidegger (1954) et Foucault (1975) mettent en avant le processus d'asservissement de la science à l'économie. Ce processus implique l'appropriation privée du savoir. Plusieurs historiens et philosophes des sciences envisagent comme conséquence, le risque de blocage de l'activité scientifique avec notamment Latour (1999) qui a exploré certains de ces mécanismes.

Mais, l'investissement en matière de formation et de recherche demeure perçu comme un facteur essentiel à la croissance économique. C'est dans cette optique, que l'Union Européenne (conseil de Lisbonne) s'est engagée pour 2010 à faire de l'Europe, "l'économie de la connaissance la plus compétitive et la plus dynamique du monde, capable d'une croissance économique durable, accompagnée d'une amélioration quantitative et qualitative de l'emploi et d'une plus grande cohésion sociale, dans le respect de l'environnement ». Or Busquin (2005), l'un des inspirateur de Lisbonne, constate le déclin de l'empire scientifique européen. L'engagement des 15 chefs d'Etat Européens se bornerait-il à n'être qu'un simple effet d'annonce ? Le bilan à mi-parcours est plutôt décevant: "un programme trop chargé ", "une coordination insuffisante ", "des priorités divergentes " et surtout un "manque de volonté des politiques des états membres » (Rapport Kok, 2004). Pour Win Kok, l'heure n'est plus à l'autosatisfaction. La majorité des décideurs politiques ne prend pas suffisamment au sérieux ses propres déclarations sur la science et l'innovation. Il en est de même pour les dirigeants des grands groupes, qui, en raison de leurs préoccupations à court terme, orientées vers le tout financier, sont prêts à sacrifier à la première occasion, la recherche et la formation. Le savoir est alors envisagé sous un seul angle : celui de marché.

\section{L'introduction du modèle marketing dans les universités}

\section{L'université entrepreunariale et le modèle marketing}

16 Le modèle de l'université entrepreunariale se traduit par l'introduction de méthodes et d'outils de gestion issus du privé. On tente d'introduire plus de souplesse et d'autonomie dans l'organisation. Simultanément, on insiste sur la nécessité de prendre en compte les besoins des usagers. Les méthodes du marketing pénètrent le secteur public. Mais le modèle de l'entreprise est en crise et l'effet de mimétisme qu'il exerce perd son sens tout en conservant paradoxalement son attractivité. L'université adopte à son tour des méthodes et des valeurs qui ont montré dans un passé proche leurs limites face aux mutations de l'économie et à la montée du chômage. Le climat actuel de guerre 
économique induit par l'absence de création de valeur en interne par les entreprises (Stratégie Business Units développée dans les années 70) est sans doute une des conséquences de cette orientation.

L'analyse de la situation récente (de 1998 à 2006) des universités nous a conduit à mettre en évidence la naissance d'un marketing des universités qui s'est caractérisé par l'introduction massive des techniques de communication depuis environ une vingtaine d'années dans les universités françaises.

L'objectif visé par ces stratégies va consister dans un premier temps à séduire pour attirer de nouveaux étudiants. Une " course à la communication » suivra, avec l'introduction du LMD. Le but pour les universités est de se positionner dans un environnement concurrentiel européen et international. Le terme de "marketing » est compris comme synonyme d'une panoplie de techniques de communication commerciale visant à permettre une implantation sur un marché. Dans ce contexte, l'idée dominante est de parvenir à construire une image de marque. L'université doit être assimilable à une marque si elle veut être concurrentielle.

La création de marque constitue l'essentiel de la réflexion stratégique des écoles et à leur suite des universités. Elle est un préalable car elle est susceptible d'apporter reconnaissance et équivalence de valeur ajoutée. Elle est avant tout une promesse de qualité. Pour les établissements d'enseignement supérieur privés, elle est aussi en mesure de justifier un prix, de conquérir de nouveaux « clients » et de les fidéliser. L'attractivité de la marque dépendra de la création d'un univers symbolique qui favorise la naissance d'un lien affectif et émotionnel en capacité de générer confiance, et «bouche à oreille ». L'objectif est de faire partager un emblème par toute une communauté. Les réseaux d'anciens étudiants des grandes écoles françaises et des universités américaines en sont une illustration. Ils permettent de fédérer notamment les étudiants actuels et anciens autour d'un statut symbolique. La puissance de ce statut valorise une reconnaissance sociale collective et satisfait des intérêts particuliers.

En mobilisant d'importants moyens certaines universités américaines sont devenues des marques internationales. En effet 150 établissements sur environ 2000 sont parvenus à se bâtir une réputation d'excellence qui correspond à un positionnement par rapport à un environnement concurrentiel international. Elles sont devenues des repères pour tous par rapport à des chercheurs reconnus pour leurs travaux, des chefs d'entreprises ou des politiques clairvoyants... La mémorisation de la marque est entretenue dans la durée par l'utilisation d'un marketing agressif allant jusqu'à exploiter les séries télévisées américaines pour favoriser une certaine lisibilité au niveau international. La marque véhiculée est devenue un moyen d'expression de certaines valeurs pour toute une société. C'est l'engagement déclaré de former les élites, les spécialistes d'une discipline, les cadres de hauts niveaux et de les reproduire. Il convient de rappeler que ces institutions accueillent principalement les enfants et les petits-enfants des anciens élèves qui sont devenus d'importants donateurs. Comme les entreprises, les établissements d'enseignement supérieur investissent leurs revenus sur les marchés financiers et dans l'immobilier. Progressivement, en raison notamment de l'allègement de la fiscalité américaine et du contexte de privatisation, les universités publiques ont été contraintes de diversifier leurs ressources et d'adopter les mêmes pratiques que les établissements privés. La propension des universités américaines à construire des marques à l'international est forte, car les attentes en matière de formation sont les mêmes à l'échelle internationale et l'économie pousse à l'adoption d'un même standard. La 
suprématie américaine en matière de création de marque d'université est une réalité qui exerce une influence sur l'ensemble des établissements d'enseignement supérieur à l'échelle mondiale. Mais ce n'est pas un hasard.

21 Le marketing, d'origine américaine est exploité par toutes les organisations et se décline sous toutes ses formes: approche produit, approche vente, approche marché et enfin optique sociétale. Au-delà de l'importance des budgets consacrés, c'est l'état d'esprit qui est déterminant. Il semble avoir irrigué l'ensemble des strates de toutes les structures organisationnelles. L'autre facteur significatif est le degré d'engagement de l'Etat pour assurer le fonctionnement de l'organisation. Au plus les ressources en provenance de l'Etat s'amoindrissent, au plus le recours au marketing se fait sentir. Confrontés au marché de l'enseignement supérieur, à l'obligation de résultats, et au manque de moyens financiers, les formations universitaires françaises, les laboratoires et en conséquence les enseignants-chercheurs peuvent se trouver de plus en plus en concurrence et exposés au marché. Les universités françaises sont appelées à adopter, outre l'usage des techniques de communication (publicité et relations publiques), la «philosophie» globale du marketing allant jusqu'à prospecter pour trouver des financements privés. Il ne s'agit plus uniquement d'utiliser une succession d'outils de communication mais d'intégrer les objectifs de l'institution en prenant en compte les missions d'un mandat pour définir un processus d'analyse, de planification et de contrôle. Cette appropriation nécessiterait l'acquisition de l'état d'esprit marketing. L'ensemble de la gestion et des personnels sont orientés vers le marché. Les étudiants doivent être conduits à accepter de payer le prix d'un diplôme et les entreprises doivent jouer leur rôle de mécène.

\section{Les opportunités à l'introduction du modèle global du marketing}

La Commission Européenne en 2003, constatait le manque de moyens affectés à l'enseignement supérieur. Mais elle déclarait aussitôt que ce manque de ressources provient du refus de financements issus du privé. En conséquence, la Commission estime que les universités "doivent utiliser le plus efficacement possible les ressources financières limitées dont elles disposent ». Elles se doivent donc de gérer la pénurie. On peut considérer que cette absence d'ambition pour l'enseignement supérieur intervient comme une contre-réforme. Dans un monde concurrentiel, la croyance au processus de promotion sociale par la formation semble devoir laisser la place à la morosité et au pessimisme en l'absence de moyen. La désillusion et les frustrations deviennent le résultat de discours utilitaristes qui ne manquent pas de faire porter arguments et conclusions sur l'importance de la citoyenneté, du lien social, de l'éthique, du développement humain durable, de la culture...

Le diplôme est indispensable pour réussir dans la vie, c'est l'illusion méritocratique (Dubet, 2004) qui conduit à la course aux diplômes et qui entraîne une forte demande sociale d'éducation. Pour Marie Duru-Bellat (2006) « ce développement de l'éducation est alors un 'crédentilisme', c'est-à-dire une façon de défendre les dispositifs scolaires comme dispositif commode de sélection pour les emplois, qui n'obéit à aucune nécessité fonctionnelle et n'apporte nul bénéfice à la société dans son ensemble ». Mais la montée en pression de l'enseignement supérieur est inévitable et mondiale. «A long terme, la croissance et l'emploi sont avant tout conditionnés par la capacité de l'économie 
d'utiliser efficacement et d'élargir simultanément le stock de connaissances scientifiques et techniques. Le savoir en sa qualité d'intrant comme de produit, est au centre du processus de croissance et de création d'emploi » (OCDE, 1996).

La massification de l'enseignement supérieur s'accompagne d'une forte mobilité internationale « destinée à équilibrer le marché du travail des personnels scientifiques et techniques " (rapport Saraswati, 2005). La mobilité internationale contribue à l'amélioration de la compétitivité et au renforcement de l'influence des pays. Elle est également essentielle pour le dynamisme des systèmes d'enseignement supérieur et de recherche (rapport Saraswati, 2005). Mais la conjugaison des deux phénomènes, course aux diplômes et mobilité internationale, conduit à s'interroger sur l'attractivité de la France et sur les modalités de financement de cette attractivité. Cela pose nécessairement des problèmes d'organisation, d'accueil, de qualité d'offre de formation, de fuite des cerveaux et donc de financement. Le ministère de l'Education nationale conclut dans une note d'information (2002), «la tendance à l'évolution des niveaux de diplôme devra se poursuivre si l'on veut coller au plus près aux besoins des entreprises » d'ici à 2010 .

Face à des contribuables qui exigent de plus en plus des comptes sur l'efficacité et l'efficience des dispositifs engagés, d'autres sources de financement pour l'enseignement supérieur doivent être envisagées : frais d'inscription, recherche de partenariats pour la recherche... Ces alternatives suivent leur chemin dans l'opinion publique avec l'idée persistante que ce qui coûte peu est sans valeur. Mais pour l'instant, la quête de nouvelles ressources se traduit surtout par la volonté de vouloir accueillir sans cesse de nouveaux étudiants. L'impact de ces exportations «invisibles " est important sur le plan économique (OCDE, 2003). Mais l'inscription de nouveaux étudiants entraînent nécessairement des investissements qui méritent de nouveaux financements. C'est le problème de la productivité des universités qui est posée. Dans nombre de pays, les étudiants contribuent aux financements de leurs études. La logique est simple: pour accéder à un enseignement de qualité il faut accepter que les bénéficiaires en paient le prix. Plusieurs idées sont avancées dont l'accord de bourses aux mérites et l'emprunt. Les étudiants acquittent « des droits par prélèvement sur le revenu de leurs parents et sur leur propre revenu à venir dans le cadre d'un programme de prêts ou d'une taxe sur les diplômes » (Banque mondiale, 1995).

Autre piste envisagée, l'industrialisation de l'éducation via les TICE. Elles facilitent la transmission des savoirs en permettant de proposer un "produit élaboré » à moindre coût capable de satisfaire le plus grand nombre et susceptible de « créer les conditions de pérennité d'un marché commercial pour les contenus de l'apprentissage en ligne et leur développement (...)» (Communiqué de la Commission du 25 avril 2002). En conséquence, la disparition physique de l'université au profit d'une structure virtuelle est aussi une alternative. Par ailleurs, les cadres et les techniciens supérieurs sont à la recherche d'enseignements professionnels pointus et de hauts niveaux capables de leur apporter une plus value sur le marché du travail. Le développement de la « formation tout au long de la vie » (notion centrale pour l'OCDE) constitue un marché qui offre des opportunités prévisibles. Les jeunes et les actifs seront prêts à investir en rapport avec la qualité des enseignements et des services offerts. Mais par ailleurs, la rentabilité de l'enseignement supérieur alimente de nombreux débats, puisque les électriciens, les plombiers et les maçons seraient recherchés à 1500 euros par mois alors qu'un niveau bac plus cinq éprouveraient des difficultés à trouver un emploi. Il gagnerait le SMIC et moins en restant longtemps stagiaire pour éviter d'être au chômage. Ainsi l'investissement devenant moins 
rentable on imagine une certaine désaffection pour la poursuite d'études. C'est la loi de l'offre et de la demande qui devrait dans les faits rétablir les équilibres.

Mais le fondement premier de la richesse des nations est l'intelligence. L'innovation permet d'assurer la compétitivité aux entreprises surtout lorsqu'elles supportent des coûts salariaux importants. Observateurs et hommes politiques effectuent régulièrement des analyses comparatives sur les efforts des recherches des nations. Invariablement le retard pris par l'Europe et la France dans les technologies de pointe et les technologies de l'information et de la communication est mis en exergue. Les classements internationaux mettent en évidence des universités françaises en perte de vitesse sur le plan de la recherche. En conséquence, il s'agit pour survivre dans un environnement compétitif mondial de se doter d'un niveau de formation amélioré et d'une recherche plus performante. Faute de quoi et pour résumer, il est souhaitable de maintenir ou de développer d'autres secteurs susceptibles d'accueillir des salariés (emplois de proximité par exemple). Autre solution: le tertiaire devrait être en mesure de créer les millions d'emplois qualifiés pour compenser avec les restructurations, les délocalisations ou la dématérialisation de l'économie. Mais l'innovation demeure encore le seul moyen d'échapper à la concurrence et constitue le véritable moteur du changement économique et social (Schumpeter, 1912). Or Aitken (1976) a formulé l'hypothèse que l'innovation est issue d'un processus de combinaisons et de recombinaisons d'informations de plus en plus denses. L'étincelle créative provient de ce flux d'information. Elle est le facteur déclencheur de l'innovation. De nombreuses études ont montré que la provenance de l'injection des fonds avait une incidence sur le résultat. Une même recherche n'aura pas le même résultat selon que les fonds proviennent de l'Etat, de mécènes, de fondations ou d'entreprises. La productivité émane principalement de fonds en provenance des entreprises.

En conséquence, l'explication des mauvaises performances françaises réside, dans l'organisation de la formation et de la recherche, dans la vision stratégique des entreprises (leur capacité à anticiper la demande et à collaborer avec des partenaires) et dans le manque de motivation, car finalement les connaissances et savoir-faire sont largement disponibles. L'émergence des pôles de compétitivité et des pôles de recherche et d'enseignement supérieur (PRES, loi de programme du 18 avril 2006 pour la recherche) intervient en réponse à ces quelques constats en alliant recherche universitaire, laboratoires privés et formation des établissements d'enseignement supérieur sur le modèle de la Silicon Valley.

Il a été démontré que le marketing fonctionnait efficacement à la condition d'être inscrit dans un système d'économie libérale de recherche du profit. Pour qu'il permette à l'organisation de progresser, il doit mesurer un retour de la part du marché (échec ou réussite). Il est alors inutile d'engager une stratégie de conquête de marché sans prendre en compte la notion de rentabilité. Or la conception d'une offre de formation orientée client et sa mise en marché nécessitent des investissements dans la durée (temps long de la formation avec risque d'obsolescence rapide) qui bornerait le marketing à une fonction essentiellement dépensière.

En effet, le marketing se définit avec un objectif ultime de satisfaction des besoins des consommateurs avec la prise en compte de leurs désirs. «Les besoins correspondent à des éléments nécessaires à la survie (...). L'individu a également fortement besoin de se divertir, de s'éduquer et de s'amuser. Ces besoins deviennent des désirs lorsque leur correspondent des objets spécifiques» (Kotler, Dubois, 2000). Mais l'université, compte 
tenu de ses missions essentielles de transmission et de production de savoir, ne peut appliquer à la lettre les principes d'un tel modèle. Il s'agirait de déclarer ouvertement que l'offre de formation peut être inadaptée à la demande. Inévitablement l'offre et la demande peuvent poursuivre des trajectoires très divergentes car les impératifs des deux logiques sont nécessairement différents : celui d'obtenir un diplôme avec un retour sur investissement immédiat en matière d'emploi pour les étudiants et pour l'université, celui de la construction des savoirs de façon désintéressée. Par ailleurs, si l'on considère que l'université est parvenue à une véritable crise de croissance, la conjoncture actuelle ne fait qu'amplifier les effets pervers en privilégiant uniquement la mise en avant de la fonction utilitariste de la formation et de la recherche et dans ce cas, l'université méconnaît pour partie sa vocation. Alain Renaud (2002) insiste sur la crise actuelle qui « peut être mortelle » car elle «met en cause les raisons d'être de l'institution ». Les élites sont en effet recrutées depuis deux siècles hors du cadre universitaire. «Cette crise de finalité est aussi une crise d'identité : qu'est-ce qu'une institution qui ne connaît pas elle-même ses fonctions? Elle induit une crise de pilotage : comment conduire, de fait, une institution dont on ne cerne pas les objectifs? ( (Renaud, 2002). Or, la capacité théorique et méthodologique du marketing à agir dans un environnement incertain et conflictuel est limitée en raison de l'aspect normalisé de ses procédures d'action et de son raisonnement linéaire de type cause (un problème à résoudre) et effet visé (transformation de la situation de d'origine).

\section{Le modèle marketing confronté à ses propres contradictions}

31 Les efforts consentis par les universités françaises en matière de marketing portent essentiellement sur la communication (publicité, relations publiques). L'objectif est de promouvoir un modèle de réussite de l'université. Les étudiants effectuent un brillant parcours dans un environnement stimulant. Ils bénéficient d'un enseignement de qualité assuré par des enseignants motivés. Les équipements et le matériel sont performants. La participation aux activités culturelles, sportives et associatives est enrichissante. Cette image idyllique véhiculée par la communication se heurte à la paupérisation de l'université et aux nombreuses accusations portées aux systèmes (sélection par l'échec, formations inadaptées au marché de l'emploi, hiérarchie entre établissements, démoralisation des enseignants, manque d'encadrement, ...).

Cette tendance à la perte de prestige est largement relayée par les médias, ce qui annule les efforts consentis en matière de communication par les établissements. Cette situation semble être propre aux universités car elle ne s'applique pas aux autres structures de l'enseignement supérieur. Les écoles sont épargnées, les médias se contentent de vanter leurs mérites notamment à l'occasion de l'organisation de pseudo événements. Les constats permanents de dysfonctionnement et de réformes absolument nécessaires pour moderniser le système d'enseignement supérieur prolifèrent. Ils ne concernent souvent que les universités comme si le dualisme (écoles et universités) qui caractérise si bien le système français n'avait aucune incidence ( $30 \%$ en moyenne du budget de l'enseignement supérieur est affecté à $4 \%$ du total des étudiants qui fréquentent les préparations aux grandes écoles et les grandes écoles). Or, l'opinion se façonne une vision de l'université conforme à celle véhiculée dans les médias. Les efforts de communication ramenés au niveau d'un établissement semblent alors bien modestes. Les messages trop élogieux 
distillés au milieu du vacarme médiatique sont trop éloignés de la réalité quotidienne et du vécu des étudiants. Ils ne peuvent parvenir à contrecarrer le sentiment de crise ambiant. Ainsi, limiter la communication à une promesse de réussite tend à inscrire le modèle de la communication marketing dans la symbolique du modèle de la poubelle. En faisant abstraction des problèmes à résoudre, la promesse fait office de décision. La logique qui consiste à idéaliser l'université est une sorte de réponse préétablie à ce qu'elle devrait être. La communication est présentée comme un cercle vertueux, capable de créer une image de marque, de lutter contre la concurrence, de convaincre et de générer lien social et motivation, ... Ses règles obéissent à une méthodologie définie et à des actions mesurables qui ont les caractéristiques d'une rationalisation créée a posteriori. Constituée d'un ensemble de techniques directement opérationnelles, la communication marketing a réponse à tous les problèmes. Elle constitue une argumentation susceptible de les régler. Mais les opérations et les messages sont reconduits alors que peu d'universités effectuent un contrôle sur leurs résultats (enquête, 2002).

Les services communication répondent aux sollicitations diverses et variées des acteurs. Les spécificités de l'organisation universitaire limitent par ailleurs la fonction de porteparole du communicant. Il peut faire l'objet d'une remise en question de la part notamment des membres du corps enseignants qui disposent d'une grande liberté de parole. Tout le monde a l'opportunité d'exprimer son point de vue et les journalistes notamment se servent nécessairement des multiples contradictions pour alimenter une polémique existante ou en créer une. Finalement, on peut s'interroger sur la marge de manœuvre des responsables de communication. Ils se trouvent confrontés à un réseau complexe d'acteurs (président, secrétaire général, vice-présidents, chargés de missions, directeurs d'unités, de laboratoires et de formations, membres des conseils, chefs de services ...) qui vont influencer et contribuer à la prise de décision. Dans ce contexte, les décisions seront la résultante de rationalités et de désirs multiples. Mais il n'y a pas toujours choix véritable et prise de décision. Alors la tentation est grande de s'inspirer de ce qui s'est déjà fait ou de ce qui se fait ailleurs. L'amont du processus stratégique de communication est négligé (veille, diagnostic). L'approche de la communication est alors plus intuitive que stratégique et dépend de la capacité du responsable de communication à argumenter des choix issus de ses propres perceptions. "Sans doute ce savoir relationnel est-il bien davantage l'expression de qualités personnelles intrinsèques conformes à un code général de cérémonie, une éthique managériale réglementant les usages communicationnels. Le discours du faire faire, du faire coopérer, si l'on est manager, puise sa force performative de la croyance partagée par tous les acteurs en la préexistence incontournable de ce code " (Gramaccia, 1999). Certes la création d'un climat de confiance entre les acteurs en interne qui nécessite de grandes qualités relationnelles personnelles, va devenir une priorité pour permettre de coordonner les échanges avec l'externe, ce qui requiert la construction d'un bon réseau relationnel.

Mais cette approche à la fois intuitive, relationnelle et conjoncturelle est-elle suffisante face à des enseignants chercheurs en quête d'une identité professionnelle à remodeler et à valoriser, des personnels IATOS préoccupés par leurs effectifs et la défense de leur statut, des étudiants focalisés par leur devenir professionnel et qui s'investissent peu dans la vie de l'établissement. Le caractère morcelé de la mission des enseignants (formation, recherche, administration, encadrement des étudiants et de personnel, définition et création de diplômes, gestion des stages, recherche de partenariat, ...), incite à une participation peu active aux instances de décision. Le faible niveau de rémunération 
conforte l'impression d'une absence de reconnaissance de la société envers ses enseignants. Pire, l'université ne serait plus un lieu protégé où l'on apprend à penser, à être créatif, responsable, critique... Le savoir deviendrait « un produit de consommation courante " et l'université serait un hypermarché. Les universitaires seraient appelés à devenir des producteurs et des marchands de connaissances et de technologies. L'information envahirait notre quotidien et se substituerait aux savoirs. La recherche serait perçue comme une activité à haut risque. Elle serait dépensière par rapport à des critères dits d'utilité... Le savoir et sa construction sont en cause. La recherche comme la formation connaissent un problème de perception.

A défaut de solutions globales, les universités en devenant ouvertes sur le monde sont devenues visibles et sujettes à polémiques. Leur crédibilité et leur prestige sont discutés ce qui conduit, paradoxalement à les fragiliser et à produire des changements. Mais, la multiplication des classements nationaux et internationaux, qui constitue un puissant vecteur de promotion, fait progresser l'idée de concurrence entre établissements. Tous souhaitent figurer parmi les meilleures places même si cette technique s'inscrit dans la pure logique élitiste américaine. Dès 1980, les journaux américains suivis par les journaux anglais affichent une hiérarchie entre établissements et en priorité entre les business schools. Les critères retenus sont variables au cours du temps et d'un palmarès à un autre. Au niveau international, les classements sont beaucoup moins nombreux à l'exception de celui établi par l'université de Jiatong à Shanghaï, en situation de quasimonopole. Ce classement retient pour critère le nombre de prix Nobel ou de médaille Fields, le nombre d'enseignants figurant parmi les auteurs les plus cités dans les revues scientifiques, le nombre d'articles publiés dans Nature et Sciences, le nombre de citations dans les index scientifiques et enfin pour une petite part la taille de l'établissement. Mais les universités de grande taille avec une recherche en sciences exactes importantes se trouvent favorisées. Au plan national, de nombreux palmarès basés sur du déclaratif cohabitent avec des méthodologies et des critères différents qui conduisent à une information incomplète et biaisée. L'impact de ces méthodes présente un danger certain car il incite à mettre en œuvre des stratégies pour remplir des questionnaires et pour créer des liens avec les agences de notation afin de gagner quelques places dans une hiérarchie pas forcément fondée. Magazines et quotidiens régionaux réalisent également, des numéros spéciaux ou des suppléments pour aider le lecteur dans ses choix. Le but de ces supports est avant tout de générer des recettes publicitaires. La probabilité de bénéficier d'une présence rédactionnelle reste liée à l'achat d'un espace publicitaire. Le lecteur peut en conséquence être induit au moins partiellement en erreur si bien que la prolifération de suppléments, de rubriques et de classements devient un élément d'opacité et d'inégalité supplémentaire. Le bon usage de ses multiples classements et conseils tronqués pour ne pas dire truqués, implique des compétences très inégalement acquises par les lycéens, les étudiants et leurs familles.

Par ailleurs, la mise en avant permanente dans le discours commun de l'opérationnalité immédiate des diplômés renforce paradoxalement l'idée d'un effort croissant à consentir par la collectivité pour l'emploi des jeunes et non en direction des universités qui assurent pourtant la formation de l'immense majorité des bacheliers. On ne peut, en effet, tout attendre de l'université. Le monde du travail doit également avoir conscience des impératifs et contraintes de l'organisation des enseignements surtout si l'on analyse la formation dans sa fonction uniquement utilitariste. Dans ce cadre, elle profite en priorité aux entreprises. Les mesures prises en faveur de l'emploi des jeunes (exonérations 
complètes ou partielles des charges, assouplissement des règles du droit au travail) et l'augmentation progressive de la durée des stages permettent aux employeurs de bénéficier d'un vivier de compétences à faibles coûts. Le monde du travail mériterait, sans doute de devenir un peu plus attractif aux yeux des étudiants. On ne peut pas prétendre vouloir recruter que des salariés sans cesse mieux formés avec des niveaux d'études sans cesse plus élevés, sans en accepter les contreparties: des fonctions, un salaire en adéquation avec le niveau d'étude et des perspectives de carrière...La logique de détournement qui consiste à réclamer l'adéquation des formations à l'emploi et à promouvoir les métiers manuels pose interrogation puisqu'elle s'accompagne finalement d'une baisse des salaires et de la précarité de l'emploi pour tous. Comment dans ces conditions motiver des jeunes, qui ne voient dans la formation, qu'un passeport pour l'emploi assujetti à des barrières, de plus en plus difficile à franchir. Face à l'incertitude du lendemain, la tentation est grande du cumul de diplômes pour certains et à l'opposé, du défaut de formation pour les autres. Marie Duru-Bellat (2006) s'est penchée sur les déceptions et les sentiments de déclassement que creuse «l'inflation scolaire ». "Si la méritocratie fonctionne ainsi comme un piège redoutable pour l'éducation elle-même, elle est également un piège pour les élèves : tous les apprentissages sont subordonnés à la pression de l'utile, avec l'ombre angoissante du marché du travail. »

L'obsession d'image des entreprises mériterait d'intégrer réellement le sens des responsabilités et l'éthique qu'elles ne manquent pas de mettre en avant dans le cadre de leurs politiques de communication. L'intégration du concept de responsabilité sociale dans la communication ne peut s'envisager sans des actes et la réciprocité des échanges entre entreprises et universités. L'établissement de liens avec les médias, les agences de notation, les entreprises et les réseaux influents sont une réelle nécessité car il est effectivement souhaitable d'éviter et d'anticiper les risques de dérives. Comme il est nécessaire de s'intéresser autant au processus, aux techniques qu'au contenu si l'on veut éviter de "communiquer parce qu'il faut communiquer " ou de "communiquer parce qu'il y a un problème de communication ». Mais la question la plus délicate pour le marketing touche à la résistance manifeste d'une partie du personnel à son utilisation et une structure organisationnelle qui impose des limites à son introduction globale.

\section{Conclusion}

Nos enquêtes auprès des communicants des universités nous ont montré que leur rôle était limité en raison d'un contexte organisationnel «stationnaire " marqué par une tendance à entretenir le mythe du secret et de la rumeur, un manque de réactivité face aux sollicitations extérieures, un processus décisionnel lent et des décisions soumises à de nombreuses critiques. L'université apparaît encore comme une organisation éclatée en raison d'une tradition individualiste et critique importante même si les instances décisionnelles se sont dépolitisées, selon Christine Musselin (2001), à partir des années 1980. La structure est dominée par des valeurs présupposées communes malgré la persistance d'un fort sentiment d'indépendance par rapport à l'établissement, en particulier chez le personnel enseignant. La hiérarchie est marquée par des pouvoirs éclatés et des centres de décisions multiples à l'intérieur de l'institution. Elle doit composer également avec les différents conseils et notamment avec un conseil d'administration qui peut jouer le rôle de contre-pouvoir. Pourtant, selon Mignot-Gérard (2003), les universités au plan local ont su développer un style de gouvernement qui 
«leur est propre venant ainsi remettre en cause l'idée largement répandue de l'uniformité du système d'enseignement supérieur français ». Pour l'auteur, «la dimension rationnelle du gouvernement des universités " est désormais mise en évidence.

Pourtant, l'insistance à promouvoir une logique entrepreneuriale, évoque le spectre d'une organisation mécaniste qui fonctionne avec un ordre et des procédures établis et dont les préoccupations sont dominées par une vision à court terme. Cette perspective d'immédiateté fait craindre une propension forte à négliger les investissements lourds et les recrutements nécessaires au développement de la recherche et de l'enseignement. Tout le monde semble aujourd'hui, s'accorder sur l'impérieuse nécessité de moderniser le système. Mais le refus de la prise en compte des particularismes générés au cours des siècles, occulte la prise en compte des facteurs de blocages inhérents à l'introduction d'une logique purement économique. L'insistance à étendre la vision rationnelle à la formation n'est-elle pas finalement un moyen efficace de se protéger de la confrontation avec la jeunesse, elle-même essentiellement préoccupée par son insertion professionnelle? L'organisation universitaire, marquée par de nombreuses contradictions, a pourtant été capable de produire de nombreux changements. « Et s'il est vrai que les universités constituent des organisations particulières, il n'en reste pas moins qu'elles existent en tant que cadre institutionnel et que le construit organisationnel et politique qui les sous-tend a un impact sur leurs capacités de décision, d'action et de changement » (Friedberg, Musselin, 1992). Vouloir maîtriser toutes les variables d'une organisation aussi complexe que l'université semble bien illusoire. A leur naissance, les universités étaient des organisations fédératrices. Elles le demeurent tout en mêlant les modèles collégial, politique, bureaucratique, de l'anarchie organisée et entrepreneurial. Le champ des interprétations devient alors inépuisable car chaque entité se caractérise par une identité distinctive.

Mais l'université dans son ensemble, évolue et se transforme tout en restant attachée à des valeurs traditionnelles et à sa fonction de transmission d'un capital culturel. Elle s'internationalise et devient virtuelle. C'est le sens même de son action qui est aujourd'hui questionné. « Construire un sens partagé » est le rôle catalyseur que pourrait jouer la communication si elle parvenait à se préserver de la futilité anti-intellectuelle du modèle marketing de la communication et du langage universitaire élitiste. Mais comment imaginer une communication porteuse de sens, sans aborder les questions fondamentales du rôle de la science, de la technique, de la culture et du statut de l'éducation, dans un monde dominé par le marché et ses incertitudes?

\section{BIBLIOGRAPHIE}

Aitken, S., The origin of radio, Princeton University Press, 1976.

Baldridge J.V., Power and Conflict in University, New York, 1971.

Banque mondiale, Priorités et stratégies pour l'éducation. Washington, 1995. 
Busquin, P. et Louis F., Le déclin de l'Empire scientifique européen. Comment enrayer la chute? éd. Luc Pire, Bruxelles, 2005.

Charle C., Verger J., Histoire des Universités, Que sais-je, Paris, 1994.

Clark B. R., The Highter Education System. Academic Organization in Cross-National Perspective. University of California Press, 1983.

Clark B. R., Creating Entrepreunarial Universities: organisationnal pathways of transformation, New York, Elsivier, 1998.

Cohen M. D., March J. G. et Olsen J. P., « A Garbage Can model of Organisationnel Choice », Administrative Science Quartely, 1972.

Cohen M. D. et March J. G., Leadership and Ambiguity; The American College President, New York, MC Graw Hill Book Company, 1974.

Crozier M., Le phénomène bureaucratique, Seuil, Paris, 1963.

Crozier M., Friedberg E., L'acteur et le système, Le Seuil, Paris, 1977.

Currie J., Vidovitch L., Micro-Economic Reform Through Managerialism in American and Australian Universities, in Universities and Globalization. Critical Perspectives, Currie and Newson, California, Sage, 1998.

Dill D. et Sporn B., Emerging Patterns of Social Demand and University Reform : Through and Glass Darkly, Pergamon, New York, 1995.

Dubet F., L'école des chances, Qu'est-ce qu'une école juste?, Seuil, Paris, 2004.

Duru-Bellat M., L'inflation scolaire. Les désillusions de la méritocratie, Seuil, Paris, 2006.

Foucaud M., Surveiller et punir, Gallimard, Paris, 1975.

Friedberg E., Musselin C., En quête d'universités. Etude comparative des universités en France et en RFA, L'Harmattan, 1989.

Goodman P., The Community of Scholars, New York, Random House, 1962.

Gouldner A. W., Parterns of Industrial Bureaucraty, Glencoe, III, Free Press, 1954.

Gramaccia G., « Communication et coopération dans l'analyse ingénierique », revue Communication et Organisation, $1^{\mathrm{er}}$ semestre, Bordeaux, 1999.

Heidegger, M., La question de la technique, essai et conférence, Gallimard, Paris, 1954.

Kok W., Relevé le défi. La stratégie de Lisbonne pour la croissance et l'emploi, office de publication des communautés européennes, Novembre, Luxembourg, 2004.

Kotler P., Dubois B., Marketing management, 10e édition, Publi-Union, Paris, 2000.

Latour, B., « Morale et technique : la fin des moyens », Réseaux, 100, 1999.

March J. G . et Simon H. A., Les organisations, publié en 1979, Dunod, Paris, 1958.

March, J. G., « Technologie de la déraison et éloge de la lenteur », propos recueillis par Jardin E., Les Organisations, Sciences Humaines, Paris, 2005.

Merton R. K., Social Theory and Social Structures, Free Press of Glencoe (1962), The Academic Community, New York, MC Graw-Hill, 1957.

Millett J. D., The Academic Community,NewYork, MC Graw Hill, 1962.

Midderlehurst R., « Changing Ledership in Universities » Open University Press, Londres, 1995. 
Mignot-Gérard S., Musselin C., Les modes de gouvernement de 37 universités françaises, Rapport d'enquête Cafi-Amue, Paris, 2000.

Mignot-Gérard S., «Le « leardership » et le « gouvernement » dans l'analyse des organisations universitaires : deux notions à déconstruire » Politique et gestion de l'enseignement supérieur, 15.2, OCDE, 2003.

Ministère de l'Eduction nationale, « Prospective emploi-formation à l'horizon 2010 ", note d'information $\mathrm{n}^{\circ} 2.30,2002$.

Musselin C., La longue marche des universités françaises, PUF, Paris, 2001.

OCDE, La stratégie de l'OCDE pour l'emploi. Technologie, productivité et création d'emploi. Volume 1. Synthèse et conclusion, 1996.

OCDE, Gestions des établissements, de nouvelles approches, 2001.

OCDE, Education, internationalisation et échanges, 2003.

Olivesi S., La communication au travail, PUG, Grenoble, 2002.

Pleffer, J., Salancik, G., « Organizational Decision Making as a Political Process », Administrative Science Quaterly, 1974.

Renaut A.,, Que faire des universités ? Bayard, Paris, 2002.

Rojot J., «Qu'est-ce que décider dans une organisation », Les Organisations, Sciences Humaines, Paris, 2005.

Schumpeter J. A., Théorie de l'évolution économique, Dalloz, Paris, 1912.

Sfez, L., Critique de la décision, Fondation Nationale des Sciences Politiques, $2^{\mathrm{e}}$ édition, 1981.

Slaughter S., Leslie L., Academic Capitalism: politics, policies and the entrepreneurial university, Johns Hopkins Press, Baltimore, 1997.

Tierney, K. E., « Symbolism and Presidential Perceptions of Leadership », in The Review of Higher Education, Volume 12, $\mathrm{n}^{\circ} 2,1989$.

Weik K. E., « Educational Organizations as Loosely Coupled Systems », Administrative Science Quaterly, 1976.

Weber M., Economie et société, Plon, Paris, 1971.

\section{RÉSUMÉS}

Les universités constituent des entités structurantes de la vie sociale, cultuelle et économique. Elles sont interpellées par les exigences de la société mondiale, la compétitivité des entreprises, les besoins des étudiants et la restriction des moyens. Quels sont les modèles organisationnels développés par les théories des organisations et du management? Pourquoi le modèle « entrepreneurial » tend à s'imposer et avec quelles limites? Quelles sont les conséquences de l'introduction du modèle marketing de la communication si l'on considère d'une part, le temps long de la recherche et de la formation et d'autre part, un contexte oscillant entre continuités et fragilités?

Universities constitute structural entities of social, cultural and economic life. They are challenged by the demands of society, the competition of companies, students' needs and financial restrictions. What are the models of organization developed by the theories of 
organization and management? Why does the "entrepreneurial" model tend to impose itself and to what extent? What are the consequences of the introduction of the marketing model of communication, if we consider, on the one hand, the long length of time for research and training and,on the other hand, a context that fluctuates between continuity and fragility.

INDEX

Mots-clés : communication, crise, décision, entrepreneurial, marketing, modèle, organisation, stratégie, université

\section{AUTEUR}

LUCIA GRANGET

Mail : granget@univ-tln.fr 